\title{
Aesthetic outcomes after surgical repair of pectus excavatum in females: Differences between patients and professional evaluators
}

\author{
Tanja Wachter ${ }^{1}$, Barbara Del Frari ${ }^{1}$, Michael Edlinger ${ }^{2}$, Evi Maria Morandi ${ }^{1}$, Christina Mayerl ${ }^{3}$, \\ Ralph Verstappen ${ }^{1}$, Emre Celep ${ }^{1}$, Gabriel Djedovic ${ }^{1}$, Johann Kinzl ${ }^{4}$, Anton Herbert Schwabegger ${ }^{1}$, \\ Dolores Wolfram ${ }^{1}$ \\ Departments of ${ }^{1}$ Plastic, Reconstructive and Aesthetic Surgery, ${ }^{2}$ Medical Statistics, Informatics and Health Economics, ${ }^{3}$ Internal Medicine III, \\ Cardiology and Angiology, and ${ }^{4}$ Psychiatry, Psychotherapy and Psychosomatic, Medical University of Innsbruck, Innsbruck, Austria
}

Background Pectus excavatum is less common in females than in males, and it often aggravates a coexistent breast asymmetry. We conducted a study comparing female patients' versus medical professionals' evaluation of pectus excavatum repair to assess differences in aesthetic outcome ratings. Moreover, we evaluated the influence of surgical correction on patients' self-perception.

Methods 0 f 30 female patients who were initially screened, 18 patients (mean age, 20 years) who underwent bar removal after surgical correction of pectus excavatum deformity participated in the survey $(60 \%)$. They completed a questionnaire rating their appearance before and after surgery and responded to a psychological questionnaire about the changes that they had experienced. The mean interval between pectus bar removal and evaluation was 28 months. Standardized preoperative and postoperative patient photographs were evaluated using the same questionnaire by a panel of medical professionals and students $(n=24)$ and the results were compared.

Results Patients rated their preoperative deformity as more severe than the other evaluators, revealing the significant impact of the deformity on patients' self-perception. Postoperatively, patient and professional evaluations were much better than before and were very similar. The psychological evaluation showed a clear improvement in well-being. The ratings of the medical professionals were not influenced by their degree of medical education.

Conclusions Surgical correction of pectus excavatum in female patients positively influences body perception and psychological well-being. It should therefore not be considered as a merely aesthetic correction, but as an important procedure to restore a patient's self-perception.

Keywords Funnel chest / Thoracic surgical procedures / Female / Self concept
Correspondence: Dolores Wolfram Department of Plastic, Reconstructive and Aesthetic Surgery, Medical University of Innsbruck, Anichstrasse 35, Innsbruck 6020, Austria Tel: +43-512-50422731 Fax: +43-512-50422735

E-mail: dolores.wolfram@i-med.ac.at
The authors thank Philip Lichtenstein, $M D$, from the Department of Radiology, Columbia University (New York, NY, USA), a native English speaker, for assistance with editing the English language of this paper. 


\section{INTRODUCTION}

Pectus excavatum (PE) or funnel chest is a congenital chest wall deformity with an incidence of 1 in 400 live births and a maleto-female ratio of $4: 1[1,2]$. In female patients, the deformity commonly leads to a distortion of the female breast, resulting in so-called breast strabism with a diminished intermamillary distance [3]. Females with PE tend to complain about the disturbing aesthetic appearance of their breasts and not about the sternal depression, which may be partly concealed by their breast tissues. Funnel chest resulting in a severe breast deformity may stigmatize a young woman, causing embarrassment, social anxiety, and other psychological disorders [4]. Surgical correction of the deformity has been shown to improve patients' quality of life and to have positive influence on self-perception $[5,6]$.

Trying to correct the deformity by augmenting the breast with silicone implants may aggravate the strabism of the breasts by making the sternal inclination more prominent, which leads to unsatisfactory aesthetic results [3]. Instead, as we have demonstrated in previous publications, remodeling the anterior thoracic wall with retrosternal metal bar implantation by a minimally invasive repair procedure (MIRPE) [7] or by a modified approach with a semi-open technique (minor open videoendoscopically assisted repair of pectus excavatum; MOVARPE) [8] corrects the underlying deformity and leads to a marked improvement in the appearance of the breasts [3].

Aesthetic correction remains a major indication for PE repair and is the most difficult parameter to measure postoperatively. The patient's satisfaction with a procedure and the surgeon's evaluation of the postoperative results can differ $[9,10]$. The aim of this study was to investigate the association between female patients' personal outcome evaluations and the assessments made by medical professionals. Standardized photographs of patients' preoperative and postoperative aesthetic appearance were analyzed by patients and by medical professionals. Differences in the evaluation based on evaluators' sex or degree of medical education were determined. Furthermore, we assessed whether the surgical intervention had an impact on the psychosocial well-being of the female participants. To our knowledge, no publications have yet investigated quality of life, satisfaction, body image, and aesthetic results before and after bar removal with an exclusive focus on female PE patients.

\section{METHODS}

\section{Study design}

The study design was prospective and approved by the local ethics committee (approval No. UN4960). All patients signed a written informed consent form and the data were analyzed anonymously. The study group included female patients who underwent correction of PE utilizing the MIRPE technique [7] or the MOVARPE [8] technique between August 2002 and February 2015 and had the pectus bar removed. Exclusion criteria were male patients, patients who underwent corrections using other techniques, or female patients who still had the pectus bar in situ. The patients underwent a clinical examination and standardized photographic documentation before and after correction.

All patients completed two questionnaires regarding their preoperative and postoperative conditions and their psychological well-being. We used specifically constructed questionnaires focused on women to evaluate their quality of life, satisfaction, and body image. The two-step Nuss Questionnaire (Pectus Excavatum Evaluation Questionnaire), which assesses the effects of the Nuss procedure on physical and psychosocial functioning, was developed and has been applied for pediatric populations. Modifications of this instrument have been made for adult male patients [11], but it has not yet been used to evaluate female breast deformities or long-term results after the removal of the pectus bar. Both questionnaires that were administered in our study, were developed by a psychologist and clinical experts with longstanding experience with chest wall deformities.

In the first questionnaire, the patients appraised their aesthetic appearance, before surgery and after pectus bar explantation, based on photographic images (Table 1). The questions addressed aesthetic aspects of the breast, symmetry and strabism

Table 1. Questionnaire 1 (for aesthetic evaluation), preoperative and postoperative

Preoperative questionnaire

Q1: How do you rate the symmetry of the chest before the surgery?

Q2: How do you rate the symmetry of the nipples/areolas before the surgery?

Q3: How do you rate the symmetry of the breasts before the surgery?

Q4: How do you rate the extent of strabism (looking towards each other) of the nipples/areolas before the surgery?

Q5: How do you rate the extent of the funnel of the chest before the surgery?

Postoperative questionnaire

Q1: How do you rate the symmetry of the chest after the surgery?

Q2: How do you rate the symmetry of the nipples/areolas after the surgery?

Q3: How do you rate the symmetry of the breasts after the surgery?

Q4: How do you rate the extent of strabism (looking towards each other) of the nipples/areolas after the surgery?

Q5: How do you rate the position of the scars?

Q6: How do you rate the appearance of the scars?

Q7: How do you rate the extent of the funnel of the chest after the surgery?

Q8: How do you rate the appearance of the chest after the surgery?

Questionnaire for the aesthetic evaluation: appraisal of various aspects of pectus excavatum deformity and repair, based on standardized preoperative photographs, rated on a visual analogue scale ( $1=$ very poor; $100=$ very good). 
Table 2. Questionnaire 2 and results of the psychological evaluation

\begin{tabular}{|lc|}
\hline Item & $\begin{array}{c}\text { Positive rate } \\
(\%)\end{array}$ \\
\hline $\begin{array}{l}\text { Q1: I am satisfied with myself and have fewer worries } \\
\text { about myself. }\end{array}$ & 89 \\
Q2: I am calmer inside. & 67 \\
Q3: I am more cheerful. & 72 \\
Q4: I do not suffer from insecurity anymore. & 83 \\
Q5: I am more confident in my interactions with other & 56 \\
people. & 89 \\
Q6: I feel freer. & 78 \\
Q7: I am calmer and more balanced. & 61 \\
Q8: I now better know what is important to me. & 67 \\
Q9: I feel less insecure in communication with others. & 78 \\
Q10: I am no longer in a battle with myself. & \\
\hline Patients' appraisal of various aspects of their psychological postoperative health \\
on a 7-point scale from -3 (very negative) to +3 (very positive) with the \\
percentage (of $n=18$ ) of positive ratings (rating $\geq 1$ ). \\
\hline
\end{tabular}

of the nipple-areola complex (NAC), depth of the PE deformity (funnel), and scar appearance. The same photographic images of all patients were independently evaluated by a panel of 24 persons at different levels of medical training with an equal sex distribution (three male/female medical students, three male/ female residents, three male/female attending surgeons), using the same questionnaire. The evaluation by medical personnel was performed twice to assess internal consistency. The interval between the first and second evaluation was 3 months. During each session, the images were presented on a screen and the questionnaire was filled out simultaneously by all investigators.

The second patient questionnaire (questionnaire 2) was a validated standardized psychological assessment administered to patients after surgery (Table 2) that addressed changes in behavior, well-being, and lifestyle.

\section{Patients and patient images}

Between August 2002 and February 2015, 43 female patients underwent surgical correction for PE utilizing the MOVARPE technique $(n=25)$ [8], the MIRPE technique $(n=8)$ [7], custom-made silicone implants $(n=6)$, and other procedures $(n=4)$. The MIRPE technique, which was first described by Nuss, involves remodeling of the anterior chest wall by implantation of a retrosternal metal bar [7]. The MOVARPE procedure is a modification of the MIRPE technique with a semiopen approach with additional skin incisions in the inframammary fold, rib cartilage incisions, or partial resection and horizontal sternum osteotomy [8]. The MOVARPE approach was used in adult patients or adolescents with athletic dispositions and in deformities with a deep funnel and severe sternum inclination or malrotation with asymmetry, whereas in young pa- tients with a symmetrical depression the MIRPE technique was used. During the study period, 30 patients had their pectus bar removed (MOVARPE group, $n=23$; MIRPE group, $n=7$ ). The bar was removed after an average of 20 months (range, 1235 months) in the MOVARPE group and 30 months (range, 14-36 months) in the MIRPE group. Patients who did not undergo bar removal were not included in the study.

All patients underwent preoperative cardiopulmonary function tests, including spirometry and echocardiography. Nine patients (50\%) showed a functional deficit, which was the indication for surgery. Of the remaining nine patients, surgery was indicated in three for psychological reasons and in six for purely aesthetic reasons. In routine clinical practice, no postoperative cardiopulmonary evaluations were performed.

Of the 30 patients who were initially screened, 18 patients (60\%) returned the questionnaire and were included in the study. Their mean age was 20 years (range, $14-41$ years) at the time of initial surgery. The interval from pectus bar removal to the last evaluation was 28 months (range, 5-92 months). The follow-up included a clinical examination and photographic documentation in frontal, oblique, and sagittal views.

\section{Appraisal of aesthetic appearance (questionnaire 1)}

Questionnaire 1 was created by a group of clinical experts with long-term experience in the treatment of chest deformities. The experts designed the questionnaire based on published instruments regarding outcome evaluations after breast surgery, and specifically modified the questionnaire for PE deformities $[11,12]$. The questionnaire included 10 items addressing the size and form of the breast, the position and symmetry of the NAC, the position and symmetry of the thoracic wall, the degree of strabism of the NAC, and the appearance and position of the scar. The questions were answered on a visual analogue scale from 1 (very poor) to 100 (very good) (Table 1 ).

\section{Psychological appraisal (questionnaire 2)}

We modified a German-language standardized psychological test [13]. The original questionnaire consisted of 42 questions assessing the subjectively perceived status of a patient in a comparative manner. High scores signified increased relaxation, serenity, and optimism, while low scores signified increased tension, insecurity, and pessimism. A psychologist with longstanding experience with patients with chest wall deformities condensed this questionnaire to the 10 questions that were most relevant for our study.

\section{Statistical analysis}

Data are represented by the percentage of a rating or as the me- 
dian and interquartile range. In comparisons, the Wilcoxon signed-rank test for nonparametric testing of paired samples was applied. When analyzing differences between patients and professionals, the latter were grouped together with a single median score. SPSS version 19.0 (IBM Corp., Armonk, NY, USA) and MedCalc version 16.8.4 (MedCalc Software, Ostend, Belgium) were used.

\section{RESULTS}

\section{Overall impressions of preoperative aesthetic appearance}

Patients rated their preoperative aesthetic appearance more poorly than the medical professionals in all categories (Table 3, Fig. 1A). The appearance of the funnel received the worst rating from the patients (median rating, 9), compared to a median rating of 27 by the professionals. Thorax symmetry and breast symmetry were rated as 13 and 12, respectively, by the patients, compared to ratings of 42 and 37, respectively, by the professionals.

\section{Overall impressions of postoperative aesthetic appearance}

Patients rated their overall postoperative appearance as 90 on average, whereas professionals reported a rating of 72 . In all aspects, patients' ratings of their own postoperative results were slightly higher than the ratings of the medical investigators, indicating a high level of satisfaction (Table 3, Fig. 1B). Fig. 2 shows an example of a patient who rated her preoperative appearance more poorly than the medical professionals, and was in agreement with the medical personnel in her postoperative rating.

\section{Differences in medical professionals' evaluations between the first and second ratings}

There was a strong consensus between the duplicated ratings, concerning both preoperative and postoperative ratings (Table 4). In the preoperative ratings the median results for symmetry of the breast and strabism were equal between the first and second evaluations ( 37 vs. 37 and 41 vs. 41, respectively), while for the other questions the differences were minimal (preoperative question 1: 42 vs. 44 , question 2: 46 vs. 45 , question 5: 27 vs.

Table 3. Results of questionnaire 1: preoperative and postoperative evaluations by patients and medical professionals

\begin{tabular}{|c|c|c|c|}
\hline & $\begin{array}{c}\text { Patients, } \\
\text { median (IOR) }\end{array}$ & $\begin{array}{l}\text { Professionals, } \\
\text { median (IOR) }\end{array}$ & P-value \\
\hline \multicolumn{4}{|l|}{ Preoperative evaluation } \\
\hline Q1: Thorax symmetry & $13(5-21)$ & $42(24-57)$ & 0.001 \\
\hline Q2: NAC symmetry & $20(8-40)$ & $46(31-64)$ & 0.012 \\
\hline Q3: Breast symmetry & $12(4-28)$ & 37 (22-63) & 0.001 \\
\hline Q4: NAC strabism & $16(10-47)$ & $41(29-54)$ & 0.098 \\
\hline Q5: Funnel appearance & $9(3-29)$ & 27 (17-34) & 0.067 \\
\hline \multicolumn{4}{|l|}{ Postoperative evaluation } \\
\hline Q1: Thorax symmetry & 76 (69-91) & $72(61-77)$ & 0.038 \\
\hline Q2: NAC symmetry & $73(61-90)$ & 68 (58-78) & 0.276 \\
\hline Q3: Breast symmetry & 76 (62-89) & $62(52-78)$ & 0.071 \\
\hline Q4: NAC strabism & 81 (70-93) & $75(68-82)$ & 0.266 \\
\hline Q5: Scar position & 92 (78-97) & $82(75-86)$ & 0.047 \\
\hline Q6: Scar appearance & $87(71-96)$ & $79(74-84)$ & 0.085 \\
\hline Q7: Funnel appearance & $90(71-94)$ & $77(71-83)$ & 0.052 \\
\hline Q8: Overall appearance & 90 (66-95) & $72(66-79)$ & 0.112 \\
\hline \multicolumn{4}{|c|}{$\begin{array}{l}\text { Aesthetic appraisal of various aspects of pectus excavatum deformity and repair } \\
\text { by patients }(n=18) \text { and medical professionals }(n=24) \text {, rated on a visual analogue } \\
\text { scale }(1=\text { very poor; } 100=\text { very good). } \\
\text { IQR, interquartile range; NAC, nipple-areolar complex. }\end{array}$} \\
\hline
\end{tabular}

\section{Fig. 1. Results of questionnaire 1}

Preoperative (A) and postoperative (B) evaluation by patients and medical professionals. NAC, nipple-areolar complex.
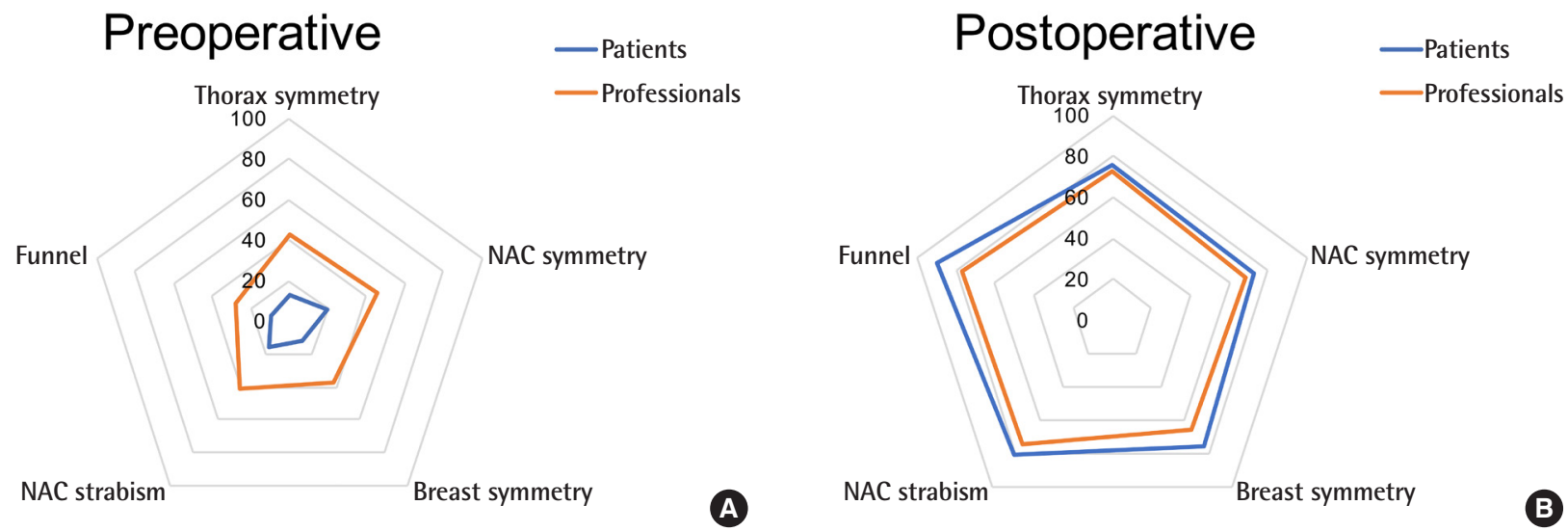


\section{Fig. 2. Pre- and postoperative standardized photo documentation}

Representative images of a patient in the preoperative (A-C) and postoperative (D-F) setting. Simultaneously with pectus bar removal, reduction mammaplasty and reconstruction of the inframammary fold was performed.
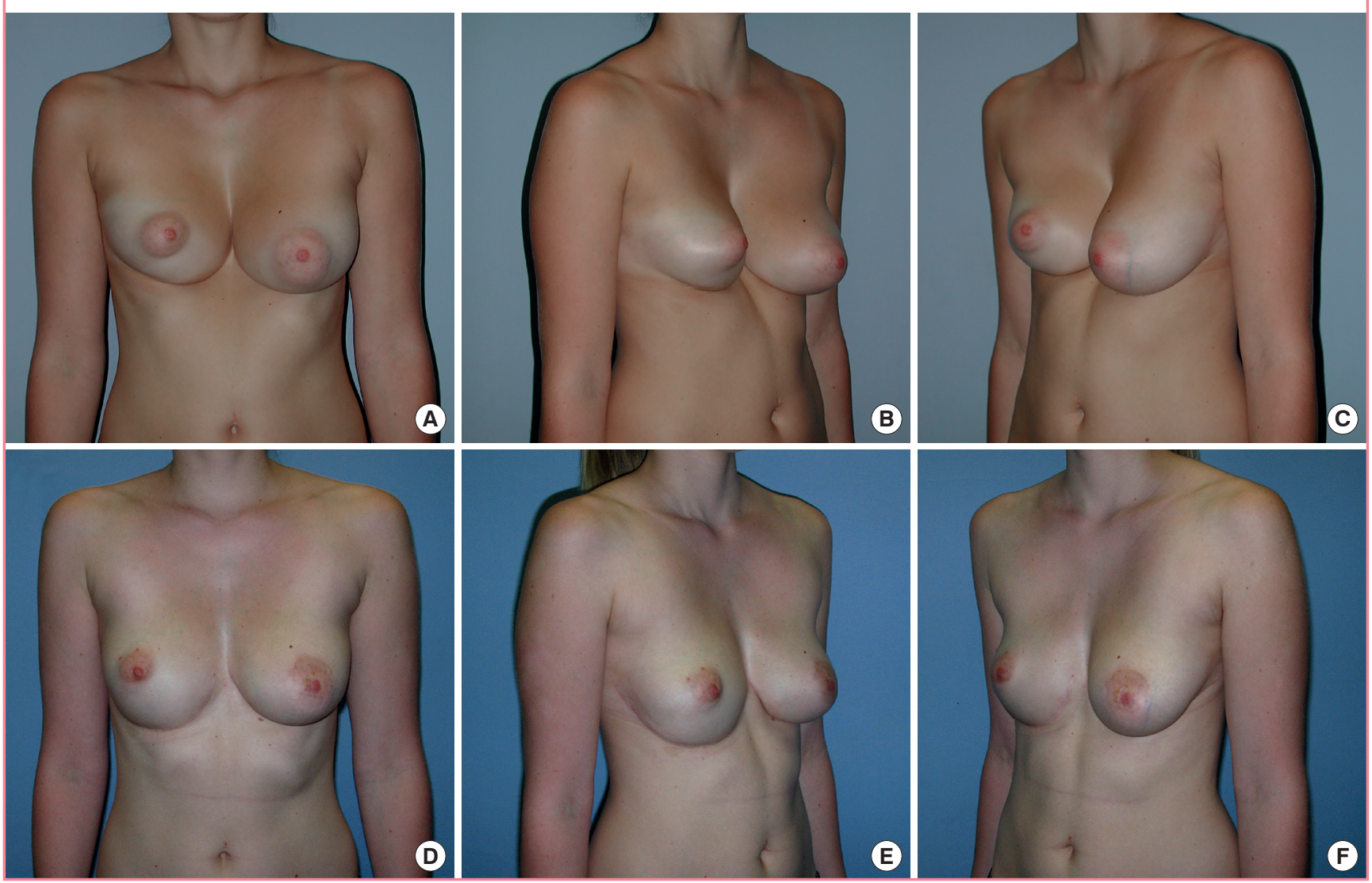

Table 4. Internal consistency based on two timely separated evaluations

\begin{tabular}{|lccc|}
\hline & \multicolumn{3}{c}{ Professionals } \\
\cline { 2 - 3 } & $\begin{array}{c}\text { First round, } \\
\text { median (IQR) }\end{array}$ & $\begin{array}{c}\text { Second round, } \\
\text { median (IOR) }\end{array}$ & \\
\hline Preoperative evaluation & & & \\
Q1: Thorax symmetry & $42(24-57)$ & $44(32-54)$ & 0.276 \\
Q2: NAC symmetry & $46(31-64)$ & $45(35-65)$ & 0.297 \\
Q3: Breast symmetry & $37(22-63)$ & $37(28-62)$ & 0.098 \\
Q4: NAC strabism & $41(29-54)$ & $41(30-52)$ & 0.266 \\
Q5: Funnel appearance & $27(17-34)$ & $29(18-37)$ & 0.383 \\
Postoperative evaluation & & & \\
Q1: Thorax symmetry & $72(61-77)$ & $74(67-77)$ & 0.037 \\
Q2: NAC symmetry & $68(58-78)$ & $69(63-76)$ & 0.050 \\
Q3: Breast symmetry & $62(52-78)$ & $69(61-78)$ & 0.002 \\
Q4: NAC strabism & $75(68-82)$ & $74(68-80)$ & 0.636 \\
Q5: Scar position & $82(75-86)$ & $80(67-85)$ & 0.485 \\
Q6: Scar appearance & $79(74-84)$ & $77(63-84)$ & 0.407 \\
Q7: Funnel appearance & $77(71-83)$ & $78(69-82)$ & 0.924 \\
Q8: Overall appearance & $72(66-79)$ & $75(68-80)$ & 0.163 \\
\hline
\end{tabular}

Replication of aesthetic appraisal of various aspects of pectus excavatum deformity and repair by medical professionals, rated on a visual analogue scale ( 1 = very poor; 100 = very good).

IQR, interquartile range; NAC, nipple-areolar complex.
29). Similar results were seen in the postoperative evaluations, with only minimal differences in median ratings.

\section{Sex differences}

There were statistically significant sex differences between the groups. Overall, male evaluators assigned lower ratings than females (Table 5).

\section{Differences in medical education}

There were only minor differences in evaluations according to the different stages of medical education. Medical students' results showed slightly better evaluations preoperatively and slightly worse evaluations postoperatively compared to all other groups.

\section{Questionnaire 2: Psychological questionnaire}

The psychological questionnaire demonstrated a clear benefit of surgical correction of PE in terms of psychological well-being. The design of the questionnaire allowed ratings of -3 to +3 , and ratings of $\geq 1$ were interpreted as indicating a positive development. Thus, $28 \%$ of patients answered 8 to 10 of the 10 ques- 


\section{Table 5. Sex differences in evaluations}

\begin{tabular}{lccr} 
& \multicolumn{2}{c}{ Professionals } & \\
\cline { 2 - 3 } & $\begin{array}{c}\text { Female, } \\
\text { median (IOR) }\end{array}$ & $\begin{array}{c}\text { Male, } \\
\text { median (IOR) }\end{array}$ & \\
\hline Preoperative evaluation & & & \\
Q1: Thorax symmetry & $38(23-51)$ & $45(34-59)$ & 0.010 \\
Q2: NAC symmetry & $50(34-65)$ & $49(30-64)$ & 0.744 \\
Q3: Breast symmetry & $35(24-61)$ & $36(21-60)$ & 0.777 \\
Q4: NAC strabism & $42(29-56)$ & $34(23-53)$ & 0.013 \\
Q5: Funnel appearance & $26(14-34)$ & $29(19-35)$ & 0.124 \\
Postoperative evaluation & & & \\
Q1: Thorax symmetry & $75(66-79)$ & $69(64-74)$ & 0.019 \\
Q2: NAC symmetry & $71(57-80)$ & $66(56-76)$ & 0.109 \\
Q3: Breast symmetry & $64(54-80)$ & $59(48-70)$ & 0.004 \\
Q4: NAC strabism & $80(75-84)$ & $69(65-79)$ & $<0.001$ \\
Q5: Scar position & $84(76-86)$ & $79(73-83)$ & 0.122 \\
Q6: Scar appearance & $81(74-87)$ & $75(69-80)$ & 0.010 \\
Q7: Funnel appearance & $79(72-84)$ & $73(66-79)$ & 0.006 \\
Q8: Overall appearance & $75(70-80)$ & $70(64-77)$ & $<0.001$ \\
\hline
\end{tabular}

Sex differences in the aesthetic appraisal of various aspects of pectus excavatum deformity and repair by medical professionals compared to patients' ratings, rated on a visual analogue scale ( $1=$ very poor; $100=$ very good).

IQR, interquartile range; NAC, nipple-areolar complex.

tions with a positive result, $39 \%$ reported positive results on 3 to 7 of the 10 questions, and $33 \%$ of patients answered only 0 to 2 of the 10 questions positively. Regarding specific questions, $89 \%$ were satisfied with themselves after surgical correction (question 1), 83\% no longer suffered from insecurity (question 4), and $78 \%$ no longer felt that they were in a battle with themselves (question 10) (Table 2).

\section{Summary of results}

In summary, the patients rated their preoperative aesthetic deformities far more poorly than the medical evaluators, whereas postoperatively, patients rated their appearance equal to or slightly better than the other evaluators. In our analysis of the evaluators, we found only minor differences in the evaluations of male versus female professionals and little impact of their degree of medical education. The psychological questionnaire demonstrated a clear improvement in well-being as a result of surgery.

\section{DISCUSSION}

In the present study, we focused on the aesthetic appearance, quality of life, and body image of female adult and adolescent patients before and after PE repair. Most previous studies concentrated on this deformity in males $[11,12,14]$ or even excluded female subjects from their studies [15]. We focused on a female cohort, since the secondary breast deformity in females aggravates their symptoms and has a pronounced effect on body image. Since PE has been shown to cause only minor functional deficits [16], insurance coverage is not always provided and the surgical correction of $\mathrm{PE}$ is sometimes still dismissed as a merely aesthetic procedure. However, many studies have clearly shown that the PE deformity has a significant influence on psychosocial development and that the benefits of surgical correction are not limited to aesthetic improvements $[11,12,14,17]$.

Our study demonstrated that female patients rated their preoperative deformities far worse than medical evaluators. Postoperatively, patients rated their appearance equal or slightly better than the medical evaluators. This indicates that the PE deformity strongly impacted patients' self-perception and that surgical correction successfully led to a self-evaluation that was in accordance with the other evaluators. We found only minor differences in the evaluations of male or female professionals and little impact of their degree of medical education.

Many reports about psychological health in PE patients have examined largely pediatric or adolescent patient series and have mainly included males $[4,11,12,17,18]$. The present study focused on both adolescent and adult female patients, ranging from 14 to 41 years of age. Studies have shown significant improvements in psychological and psychosocial well-being after PE repair in children $[5,12]$ and young adults [11], but the results of these studies were not based exclusively on females. Our psychological questionnaire confirmed the improvements in quality of life and psychological well-being after surgery, specifically in female patients. As stated previously, this is of particular importance because the PE deformity leads to an overall more severe aesthetic distortion in female patients than in male patients, as a result of the resultant breast deformity. Furthermore, previous investigations have usually evaluated patients before removal of the pectus bar $[5,11,12]$. Several investigators have pointed out that data on quality of life after bar removal were not available, although these data are needed to justify performing the Nuss or MOVARPE procedure for aesthetic reasons. So far, only three reports have included postoperative evaluations, and all of these demonstrated high patient satisfaction with the results of the Nuss procedure after removal of the pectus bar [18-20]. To our knowledge, our study is the first to evaluate female PE patients' quality of life and satisfaction after bar explantation.

Many studies on aesthetic outcomes after breast surgery have confirmed that patients and medical professionals have different perceptions of the results [10,21]. Previously, we conducted a study comparing the aesthetic evaluations of patients, medical professionals, and laypersons after implant-based breast reconstruction [9]. In oncologic patients, we showed that patients rat- 
ed their postoperative results significantly more positively than the other evaluators. Similar findings have also been reported in other studies on breast cancer patients, indicating that the differences in evaluations among patients, physicians, and laypeople are not based only on aesthetic criteria; instead, they are multifactorial [10]. These results in oncologic patients are in contrast to the results of the current study on PE repair, where only the preoperative evaluations showed different ratings between patients and the other evaluators. The postoperative evaluations yielded similar aesthetic ratings in all groups. In our view, this may confirm that PE repair corrects not only an aesthetic deformity, but also has a positive influence on patients' self-perception.

Interestingly, a study by Kim et al. [19], evaluating satisfaction after pectus bar removal in 39 children through a questionnaire, came to a different conclusion. The operative results were evaluated by the patients and their parents, as well as by two surgeons and two nurses. The surgical team's scores for overall satisfaction were higher than the patients' and/or parents' scores, and the same pattern was found for the scores for recommendation of the surgery to others. The authors suggested that preoperative expectations different among patients, parents, and the surgical team, and that a sufficient clarification should therefore be provided prior to surgery. We agree that patients and their parents should be thoroughly informed before surgery in order to ensure that they have realistic expectations regarding outcomes.

Patient satisfaction has become an important tool as a measure of the quality and outcomes of medical care [22]. A review of patients' evaluations of medical services that included results from over 50 studies showed that the average percentage of satisfied patients was about 78\% [23]. The most common assumption is that satisfaction is defined as an "evaluation based on the fulfillment of expectations" [24]. Various studies have tested this theory and have come to the conclusion that there is evidence to suggest that patients' expectations are involved in evaluations, but not in a simplistic way $[22,25]$. Expectations are modified throughout the process of care and influence evaluations. A discrepancy model has been proposed, suggesting that dissatisfaction is only expressed when an extreme negative event occurs, and that the expression of dissatisfaction is likely an indicator that something went wrong [24]. Therefore the high satisfaction rates in most studies are a questionable measure of medical quality, and the satisfaction questionnaires used in studies are still based on an insufficient knowledge of how and what patients evaluate [24].

In accordance with these points, objective measures were analyzed in our study, and differences were still present between medical evaluators and patients. This suggests that factors be- yond objective criteria influenced the evaluations.

Since self-perception is a major contributor to therapeutic decision-making, a systematic evaluation of body image should be included in the assessment of patients with thoracic wall deformities. A multidisciplinary approach, including a psychological evaluation, is mandatory for the treatment of PE deformities, and psychological screening should routinely be carried out in all patients with thoracic wall deformities. The careful selection of patients following multidisciplinary preoperative management is important for preventing disappointing results.

The limitations of this study are its retrospective data acquisition nature and the small number of patients included, which limited our ability to draw generalizable conclusions. Nonetheless, this study presents the largest experience to date, with the longest follow-up of female patients who underwent PE repair with retrosternal pectus bar implantation. Further work is needed to verify that the information the current study obtained from a small number of patients is representative of a larger population.

In summary, our results show that female patients with a PE deformity and secondary breast deformity can greatly benefit from surgical correction, with high levels of satisfaction and improvements in their quality of life. We therefore conclude that corrective surgery of $\mathrm{PE}$ has a positive effect on patients' physical and psychological well-being. Psychological improvement through aesthetic correction of the deformity is an indication for surgery.

\section{NOTES}

\section{Conflict of interest}

No potential conflict of interest relevant to this article was reported.

\section{Ethical approval}

The study was approved by the Institutional Review Board of the Medical University of Innsbruck (IRB No. UN4960) and performed in accordance with the principles of the Declaration of Helsinki. Written informed consents were obtained.

\section{Patient consent}

The patients provided written informed consent for the publication and the use of their images.

\section{Author contribution}

Conceptualization: Wolfram D, C Mayerl C, Schwabegger AH. Data curation: Morandi EM, Verstappen R, Edlinger M, Celep E. Formal analysis: Edlinger M, Wachter T. Methodology: Wol- 
fram D, Kinzl J, Del Frari B. Project administration: Wolfram D, Schwabegger AH, Djedovic G. Visualization: Morandi EM, Del Frari B. Writing original draft: Wachter T, Morandi EM, Del Frari B. Writing - review \& editing: Wolfram D, Morandi E.

\section{ORCID}

Barbara Del Frari

Michael Edlinger

https://orcid.org/0000-0002-3089-7109

Evi Maria Morandi https://orcid.org/0000-0001-8801-3268

Ralph Verstappen

https://orcid.org/0000-0001-6953-0104

Gabriel Djedovic

https://orcid.org/0000-0002-2609-109X

Anton Herbert Schwabegger

https://orcid.org/0000-0002-7253-4402

Dolores Wolfram

https://orcid.org/0000-0003-0920-7211

\section{REFERENCES}

1. Kotzot D, Schwabegger AH. Etiology of chest wall deformities: a genetic review for the treating physician. J Pediatr Surg 2009;44:2004-11.

2. Cartoski MJ, Nuss D, Goretsky MJ, et al. Classification of the dysmorphology of pectus excavatum. J Pediatr Surg 2006;41:1573-81.

3. Schwabegger AH, Del Frari B, Pierer G. Aesthetic improvement of the female breast in funnel chest deformity by surgical repair of the thoracic wall: indication or lifestyle surgery? Plast Reconstr Surg 2012;130:245e-253e.

4. Einsiedel E, Clausner A. Funnel chest: psychological and psychosomatic aspects in children, youngsters, and young adults. J Cardiovasc Surg (Torino) 1999;40:733-6.

5. Roberts J, Hayashi A, Anderson JO, et al. Quality of life of patients who have undergone the Nuss procedure for pectus excavatum: preliminary findings. J Pediatr Surg 2003;38: 779-83.

6. Dohrmann P, Lotz W, Mengel W. Psychological and cosmetic indications for the surgery of funnel chest. $Z$ Kinderchir 1987;42:286-90.

7. Nuss D, Kelly RE Jr, Croitoru DP, et al. A 10-year review of a minimally invasive technique for the correction of pectus excavatum. J Pediatr Surg 1998;33:545-52.

8. Del Frari B, Schwabegger AH. Clinical results and patient satisfaction after pectus excavatum repair using the MIRPE and MOVARPE technique in adults: 10-year experience. Plast Reconstr Surg 2013;132:1591-602.

9. Wachter T, Edlinger M, Foerg C, et al. Differences between patients and medical professionals in the evaluation of aesthetic outcome following breast reconstruction with implants. J Plast Reconstr Aesthet Surg 2014;67:1111-7.
10. Beesley H, Ullmer H, Holcombe C, et al. How patients evaluate breast reconstruction after mastectomy, and why their evaluation often differs from that of their clinicians. J Plast Reconstr Aesthet Surg 2012;65:1064-71.

11. Krasopoulos G, Dusmet M, Ladas G, et al. Nuss procedure improves the quality of life in young male adults with pectus excavatum deformity. Eur J Cardiothorac Surg 2006;29:1-5.

12. Kelly RE Jr, Cash TF, Shamberger RC, et al. Surgical repair of pectus excavatum markedly improves body image and perceived ability for physical activity: multicenter study. Pediatrics 2008;122:1218-22.

13. Zielke M, Kopf-Mehnert C. Veränderungsfragebogen des Erlebens und Verhaltens. Göttingen: Beltz Test GmbH; 1978.

14. Jacobsen EB, Thastum M, Jeppesen JH, et al. Health-related quality of life in children and adolescents undergoing surgery for pectus excavatum. Eur J Pediatr Surg 2010;20:8591.

15. Steinmann C, Krille S, Mueller A, et al. Pectus excavatum and pectus carinatum patients suffer from lower quality of life and impaired body image: a control group comparison of psychological characteristics prior to surgical correction. Eur J Cardiothorac Surg 2011;40:1138-45.

16. Lawson ML, Mellins RB, Tabangin M, et al. Impact of pectus excavatum on pulmonary function before and after repair with the Nuss procedure. J Pediatr Surg 2005;40:17480.

17. Lawson ML, Cash TF, Akers R, et al. A pilot study of the impact of surgical repair on disease-specific quality of life among patients with pectus excavatum. J Pediatr Surg 2003; 38:916-8.

18. Metzelder ML, Kuebler JF, Leonhardt J, et al. Self and parental assessment after minimally invasive repair of pectus excavatum: lasting satisfaction after bar removal. Ann Thorac Surg 2007;83:1844-9.

19. Kim HK, Shim JH, Choi KS, et al. The quality of life after bar removal in patients after the Nuss procedure for pectus excavatum. World J Surg 2011;35:1656-61.

20. Sacco Casamassima MG, Gause C, Goldstein SD, et al. Patient satisfaction after minimally invasive repair of pectus excavatum in adults: long-term results of Nuss procedure in adults. Ann Thorac Surg 2016;101:1338-45.

21. Sneeuw KC, Aaronson NK, Yarnold JR, et al. Cosmetic and functional outcomes of breast conserving treatment for early stage breast cancer. 1. Comparison of patients' ratings, observers' ratings, and objective assessments. Radiother Oncol 1992;25:153-9.

22. Williams B, Coyle J, Healy D. The meaning of patient satis- 
faction: an explanation of high reported levels. Soc Sci Med 1998;47:1351-9.

23. Lebow JL. Similarities and differences between mental health and health care evaluation studies assessing consumer satisfaction. Eval Program Plann 1983;6:237-45.
24. Williams B. Patient satisfaction: a valid concept? Soc Sci Med 1994;38:509-16.

25. Linder-Pelz S. Social psychological determinants of patient satisfaction: a test of five hypothesis. Soc Sci Med 1982;16: 583-9. 